\title{
A Space/Fast-Time Adaptive Monopulse Technique
}

\author{
Yaron Seliktar, ${ }^{1}$ Douglas B. Williams, ${ }^{2}$ and E. Jeff Holder ${ }^{3}$ \\ ${ }^{1}$ Jerusalem College of Engineering, P.O. Box 3566, Jerusalem 91035, Israel \\ ${ }^{2}$ School of Electrical and Computer Engineering, Georgia Institute of Technology, Atlanta, GA 30332-0250, USA \\ ${ }^{3}$ Sensors and Electromagnetic Applications Laboratory, Georgia Tech Research Institute, 7220 Richardson Road, Smyrna, \\ GA 30080-1041, USA
}

Received 28 October 2004; Revised 29 May 2005; Accepted 14 June 2005

\begin{abstract}
Mainbeam jamming poses a particularly difficult challenge for conventional monopulse radars. In such cases spatially adaptive processing provides some interference suppression when the target and jammer are not exactly coaligned. However, as the target angle approaches that of the jammer, mitigation performance is increasingly hampered and distortions are introduced into the resulting beam pattern. Both of these factors limit the reliability of a spatially adaptive monopulse processor. The presence of coherent multipath in the form of terrain-scattered interference (TSI), although normally considered a nuisance, can be exploited to suppress mainbeam jamming with space/fast-time processing. A method is presented offering space/fast-time monopulse processing with distortionless spatial array patterns that can achieve improved angle estimation over spatially adaptive monopulse. Performance results for the monopulse processor are obtained for mountaintop data containing a jammer and TSI, which demonstrate a dramatic improvement in performance over conventional monopulse and spatially adaptive monopulse.
\end{abstract}

Copyright $\odot 2006$ Hindawi Publishing Corporation. All rights reserved.

\section{INTRODUCTION}

A tracking radar requires a high-precision angular measurement of a target's azimuth (or elevation) which is traditionally achieved using monopulse processing. Historically, monopulse radars employed two separate feed horns on a single antenna element in order to generate two receive beams that were slightly offset in azimuth (or elevation) angle. Sum and difference outputs were formed by summing and subtracting the two beam outputs, respectively. The ratio of difference to sum output voltages, called the error voltage, was then used to determine the degree of correction necessary to realign the beam axis with the target.

With the introduction of phased array technology, it became unnecessary to employ special hardware for monopulse processing, since the array itself can electronically form the multiple beams needed. A typical conventional monopulse processor for a phased array radar is obtained by appropriately phasing the individual array channels to obtain sum and difference outputs. The ratio of difference to sum outputs provides the measure by which the angle offset from the beam axis (i.e., look direction) is determined. The updated angle measurement is then used to recompute phases for the channels so as to realign the beam axis with the target.

Mainbeam jamming occurs when the jammer signal is directly impinging on the radar's receive beam. Spatially adaptive processing works well when target and jammer are adequately separated in angle. However, as the separation diminishes until target and jammer both appear within the mainbeam, performance degrades [1]. This degradation is most severe when the target and jammer are coaligned and the spatially adaptive processor is unable to achieve any cancellation at all. The problem of using a spatially adaptive processor is further compounded in that its pattern can become very distorted in the presence of mainbeam jamming.

In $[2,3]$ it is shown that the coherent multipath commonly referred to in the radar literature as terrain-scattered interference (TSI) can be exploited to significantly improve the mitigation of interference. The operation of this processor is based on the principle that the direct path broadband jammer can be used to predict and, thus, cancel the delayed jammer reflections from the ground (i.e., TSI) present in the mainbeam. Conversely, TSI can be used to estimate and cancel the direct path jammer in the mainbeam $[2,4-6]$. In the latter case the TSI is in effect used to form a "reference beam" of the jammer, through which the jammer is cancelled. The effectiveness of adaptive TSI processing for mitigating both TSI and mainbeam jamming motivates us to investigate ways of incorporating it with monopulse processing.

Other authors have considered the development of adaptive monopulse techniques, especially for situations in which there is mainbeam jamming or clutter. For example, the author in [7] implements a minimum variance monopulse 
processor in the STAP domain to extract simultaneous direction-of-arrival (DOA) and Doppler estimates, with improvement cited by use of reduced sidelobe response (in the joint angle-Doppler domain) in suppressing clutter.

The monopulse concept is further generalized in [8] to include arbitrary parameter estimation in the STAP domain with partial preprocessing (e.g., subarray) and generalized beamforming.

The approach proposed here contrasts with those mentioned as well as others in two respects:

(i) the added dimensionality of the problem is in the fast time as opposed to slow time (classic STAP);

(ii) the added dimensionality is strictly employed in utilizing to advantage a nuisance interference, TSI, in improving DOA estimation.

The proposed approach is not offered as a competing approach to existing STAP monopulse techniques, but rather, as a treatment of the specific problem of angle estimation when either TSI alone or mainbeam jamming together with TSI corrupt the sampled returns. In such scenarios slow-time STAP by itself is of limited benefit since the sparsely spaced coherent samples offer little benefit in predicting and subsequently mitigating the direct path mainbeam jammer signal from its scattered multipath. By the same token the proposed approach depends on the presence of strong TSI in the returns, without which the incorporation of fast-time taps is of little or no benefit for mainbeam jammer cancellation.

\section{TSI PROCESSING}

A radar has to contend with many forms of interference, including jamming, clutter (ground returns), and TSI. The latter is the most complicated form of interference, and by far the most difficult to suppress. Just as the radar's transmitted signal reflects from the surrounding terrain to form a composite clutter signal at the aperture, so, too, can the jammer signal reflect from the surrounding terrain to form TSI.

Such interference may be intentional or a result of the poor sidelobe behavior that is characteristic of noise jammers. The delayed and scaled jammer reflections integrate at the aperture to form the composite TSI signal. Multipath components may be received over a wide angular extent and at varying delays potentially leading to significant spatio-temporal correlation at the array elements. This characteristic of TSI motivates the use of adaptive space/fasttime (SFT) processing as discussed below.

\subsection{Space/fast-time processing}

Considered here is a radar system that transmits a single pulse and samples the returns on an $N$-element uniform linear array. ${ }^{1}$ It collects $L$ temporal samples ${ }^{2}$ from each element

\footnotetext{
${ }^{1}$ Application to planar arrays is possible at the expense of increased dimensionality.

${ }^{2}$ In our notation, $N$ denotes a scalar constant, $\mathbf{n}$ a spatial vector, and $\mathbf{N}$ a space-time vector or matrix. The transpose of a matrix or vector quantity, $\mathbf{N}$, is denoted by $\mathbf{N}^{T}$. Similarly, Hermitian transpose is denoted by $\mathbf{N}^{H}$.
}

receiver, where each time sample corresponds to a range cell. The temporal dimension of interest here is referred to as fast time. ${ }^{3}$ The collection of samples is represented by an $N \times L$ data matrix $\mathbf{X}$ with elements $x(n, l)$. A spatial snapshot consists of $N$ elements of spatial data from the $l$ th range cell (i.e., lth column of $\mathbf{X})$ :

$$
\mathbf{x}(l)=\left[\begin{array}{llll}
x(0, l) & x(1, l) & \cdots & x(N-1, l)
\end{array}\right]^{T} .
$$

Similarly, a SFT snapshot, $\mathbf{X}(l)$, consists of $T$ consecutive spatial snapshots in descending order of time index stacked into a vector (i.e., a stack of $T$ consecutive columns of $\mathbf{X}$ in descending order of time index),

$$
\mathbf{X}(l)=\left[\begin{array}{llll}
\mathbf{x}^{T}(l) & \mathbf{x}^{T}(l-1) & \cdots & \mathbf{x}^{T}(l-T+1)
\end{array}\right]^{T} .
$$

The output of a fast-time processor, $z(l)$, is a weighted sum of the components of the SFT snapshot, $\mathbf{X}(l)$, at a given instant of time (index), $l$ :

$$
z(l)=\mathbf{W}^{H} \mathbf{X}(l)
$$

Thus, the SFT processor is described by the $N T \times 1$ weight vector $\mathbf{W}$.

The simplest type of processing that can be performed on the data is nonadaptive spatial processing (i.e., where $T=$ 1 ), also known as conventional processing. In conventional processing,

$$
\mathbf{W}=\mathbf{v}\left(v_{\ell}\right)=\left[\begin{array}{llll}
1 & e^{j 2 \pi v_{\ell}} & \cdots & e^{j 2 \pi v_{\ell}(N-1)}
\end{array}\right]^{T}
$$

is a spatial steering vector defined at spatial frequency $\nu_{\ell}=$ $(D / \lambda) \sin \left(\phi_{\ell}\right)$, where $\phi_{\ell}$ is the target's look direction, $D$ is the interelement spacing of the array, and $\lambda$ is the radar's operating wavelength [3].

\subsection{TSI mitigation}

More so than with clutter and jamming, TSI lacks a consistent and predictable structure. As such, in mitigating TSI, it is necessary to employ adaptive processing. Adaptive processing represents a more sophisticated class of processors and typically requires solving for a set of weights $\mathbf{W}$ that are optimal in the mean square sense. In other words, the mean square output of the processor,

$$
\zeta=E\left\{|z(l)|^{2}\right\}=E\left\{\mathbf{W}^{H} \mathbf{X} \mathbf{X}^{H} \mathbf{W}\right\}=\mathbf{W}^{H} \mathbf{R}_{\mathbf{X}} \mathbf{W},
$$

is minimized with respect to $\mathbf{W}$ subject to the constraints $\mathbf{C W}=\mathbf{c}$. The constraint matrix, $\mathbf{C}$, may consist of a set of space-time steering vectors, whose spatio-temporal locations indicate where the constraints are to be applied. The constraint vector, $\mathrm{c}$, contains the desired responses for signals arriving from the respective locations. The solution to the minimization problem can be expressed in closed form as

$$
\mathbf{W}=\mathbf{R}_{\mathbf{X}}^{-1} \mathbf{C}^{H}\left(\mathbf{C R}_{\mathbf{X}}^{-1} \mathbf{C}^{H}\right)^{-1} \mathbf{c} .
$$

\footnotetext{
${ }^{3}$ Fast time is distinguished from the slow time interpulse dimension encountered in Doppler processing.
} 
In processing TSI corrupted data, the constraints may be selected so as to satisfy two sets of conditions at the look direction $v_{\ell}$ : namely, unity gain at the first tap and zero gain at successive taps. The latter set of constraints are known as range constraints or null constraints. ${ }^{4}$ The constraint quantities are given as

$$
\mathbf{C}=\mathbf{I}_{T} \otimes \mathbf{v}^{H}\left(\nu_{\ell}\right), \quad \mathbf{c}=\left[\begin{array}{llll}
1 & 0 & \cdots & 0
\end{array}\right]^{T},
$$

where $\mathbf{I}_{T}$ is a $T \times T$ identity matrix, and $\otimes$ denotes a Kronecker product. The resulting set of weights have the desirable property that a target at the look direction passes through the processor undistorted, or in other words there is no target spreading.

To gain intuition into the operation of an adaptive SFT processor, consider a scenario whereby both a sidelobe jammer and TSI corrupt the returns. Furthermore, consider that the TSI spans the extent of a number of sidelobes as well as the mainbeam (not an unlikely scenario given the typical wide angular extent of TSI). Although the sidelobe jammer maybe easily mitigated through a sharp null in the sidelobe, a wide null that would occupy the entire mainbeam as well as a few sidelobes would be necessary to null the TSI in that fashion. This is problematic in that a wide null may not penetrate as deep as a narrow null, and, more importantly, the wide null spanning the mainbeam mitigates the target as well.

On the other hand, an SFT adaptive processor creates for itself a $T$ sample reference beam of the jammer. Since TSI is a composite of delayed versions of the same jammer realization, the TSI can be predicted from the reference signal and cancelled to a greater extent than the signal within the mainbeam region.

\subsection{Mainbeam jammer mitigation}

As mentioned in the introduction, SFT processing not only rids the output of TSI but, with proper choice of constraints, may also be effective at mitigating a mainbeam jammer. The principle is that TSI returns can be used to predict the temporal structure of the jammer signal, thus allowing its cancellation without resorting to placement of a spatial null in the mainbeam. A spatial null in the mainbeam can cause a target in the mainbeam to become mitigated as well. By predicting the jammer waveform through its coherent echos, nulling in the mainbeam is reduced, thus preserving target integrity, and yet suppressing the jammer.

For a thorough treatment of the algorithm the reader is referred to $[2,4-6]$.

\footnotetext{
${ }^{4}$ Range constraints are particular examples of Frost constraints [9], where Frost chooses the value of $\mathbf{c}$ to give a user-defined filter that is exact at the look direction.
}

\section{MONOPULSE}

\subsection{Conventional monopulse}

As mentioned in the introduction, a phased array monopulse processor requires the formation of sum and difference beams through the appropriate phasing of the array elements. In one method a standard steering vector is selected for the sum channel and its derivative with respect to spatial frequency for the difference channel:

$$
\mathbf{v}_{\Sigma}=\mathbf{v}\left(\nu_{\ell}\right), \quad \mathbf{v}_{\Delta}=\left.\frac{\partial \mathbf{v}(\nu)}{\partial \nu}\right|_{\nu_{\ell}} .
$$

The difference beam possesses a null at the look direction, $v_{\ell}$, and anti-symmetrical lobes on each side of the null as illustrated in Figure 1.

In a conventional (nonadaptive) configuration, the monopulse processor merely consists of two sets of weights set to the sum and difference steering vectors, respectively:

$$
\mathbf{w}_{\Sigma}=\mathbf{v}_{\Sigma}, \quad \mathbf{w}_{\Delta}=\mathbf{v}_{\Delta} .
$$

The sum and difference outputs are given in terms of the respective processors,

$$
z_{\Sigma}(l)=\mathbf{w}_{\Sigma}^{H} \mathbf{x}(l), \quad z_{\Delta}(l)=\mathbf{w}_{\Delta}^{H} \mathbf{x}(l),
$$

where $\mathbf{x}(l)$ is the $N \times 1$ spatial snapshot at time instant (index) $l$, as defined in (1).

The real part of the ratio of difference to sum outputs is known as the error voltage,

$$
\epsilon_{v}(l)=\mathfrak{R}\left\{\frac{z_{\Delta}(l)}{z_{\Sigma}(l)}\right\} .
$$

Since the array sensor outputs can be complex-valued, the ratio of beam outputs is in general complex. However, for realvalued beam patterns such as those in Figure 1, it is evident that only real-valued ratios, $z_{\Delta} / z_{\Sigma}$, correspond to a physical target, and, therefore, the imaginary part of the ratio should be discarded since it is primarily due to interference. Once an error voltage is available, it can be used in conjunction with a monopulse response curve (MRC) to arrive at an angle estimate, $\hat{\phi}$, of the target's true azimuth $\phi$.

The MRC, which plays a key role in monopulse processing, represents the ideal response of the monopulse processor (i.e., error voltage $\epsilon_{v}$ ) to targets within the mainbeam. The spatial response or beam pattern of a spatial weight vector $\mathbf{w}$ is defined by $\boldsymbol{W}(\phi)=\mathbf{w}^{H} \mathbf{v}(\phi)$. The MRC is defined as the real part of the ratio of difference to sum responses,

$$
\mathcal{M}(\phi) \equiv \mathfrak{R}\left\{\frac{\mathcal{W}_{\Delta}(\phi)}{\mathcal{W}_{\Sigma}(\phi)}\right\} .
$$

This definition is suitable when the ratio $\mathcal{W}_{\Delta}(\phi) / \mathcal{W}_{\Sigma}(\phi)$ is real-valued or predominantly real. If the ratio is imaginaryvalued or predominantly imaginary, as may happen with a particular choice of sum and difference weights, then the real part should be replaced with the imaginary part in both (11) and (12). 


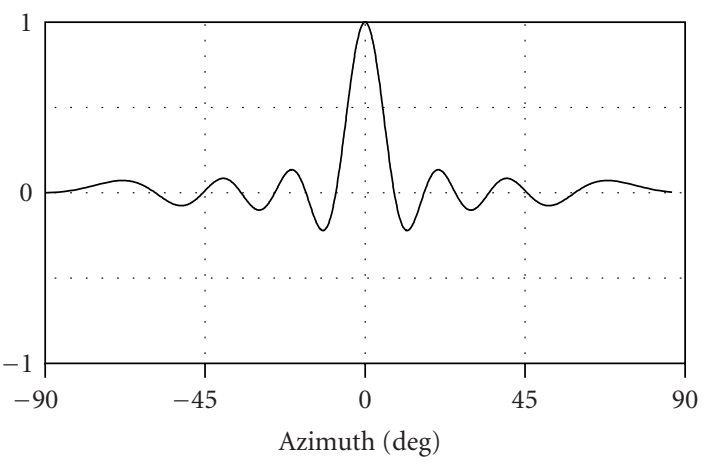

(a)

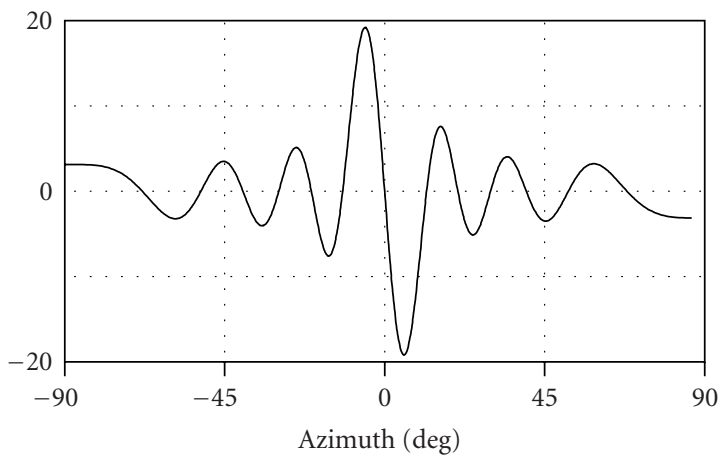

(b)

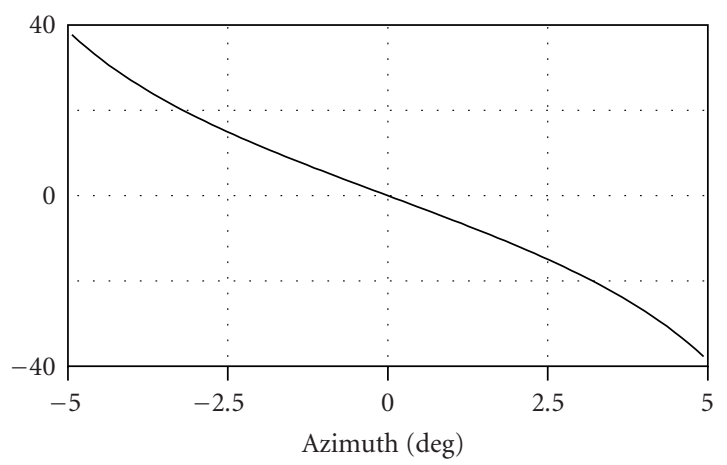

(c)

FIgURE 1: (a)-(b) Sum/difference responses and (c) corresponding monopulse response curve. Note that for illustration purposes, phase-centered weights were employed in order that the responses be purely real.

Figure 1c illustrates the response of a conventional monopulse processor to targets ranging in azimuth from $-5^{\circ}$ to $5^{\circ}$. Given an error voltage measurement, the target azimuth is determined by inverse mapping the error voltage through the MRC, $\hat{\phi}=\mathcal{M}^{-1}\left(\hat{\epsilon}_{v}\right)$, as illustrated in Figure 2.

In practice the processor pair $\mathbf{w}_{\Sigma}$ and $\mathbf{w}_{\Delta}$ are not able to completely reject interference. The residual interference that is present in the real component of $z_{\Delta} / z_{\Sigma}$ causes the measured error voltage, $\hat{\epsilon}_{v}$, to deviate from its ideal value, $\epsilon_{v}$. The corresponding error in the azimuth angle measurement, $\epsilon_{\phi}=\hat{\phi}-\phi$, is illustrated by the dashed lines in Figure 2 .

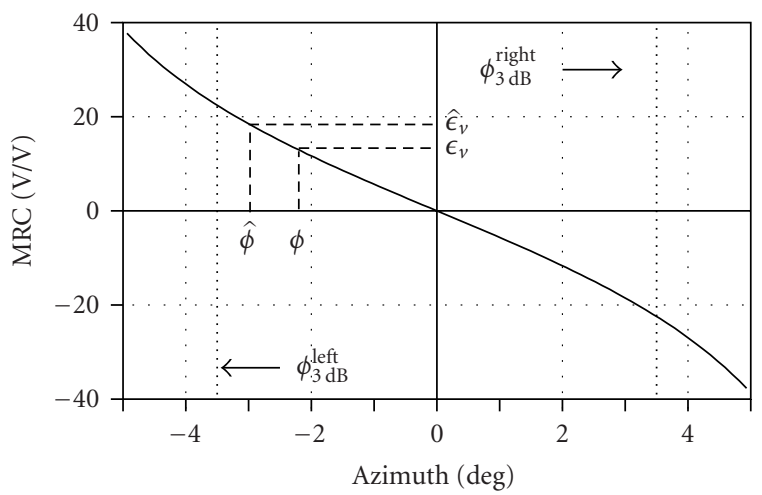

FIgURE 2: Inverse mapping an error voltage with an MRC.

\subsection{Performance evaluation}

One particularly useful performance measure for the monopulse processor is the standard deviation of the angle error (STDAE)

$$
\sigma_{\epsilon_{\phi}}=\sqrt{E\left\{\left|\epsilon_{\phi}\right|^{2}\right\}}
$$

Qualitatively, the flatter the MRC, the greater the resulting error in azimuth reading for a given deviation of error voltage. Therefore, it is desirable to have a "well-sloped" curve such as the one shown in Figure 2.

\subsection{Spatially adaptive monopulse}

A major shortcoming of conventional monopulse is that it may not provide adequate suppression of jamming and other forms of interference. Spatial adaptive monopulse has been proposed as an effective means to counter the problem of sidelobe jamming and, to a limited extent, mainbeam jamming [1]. Several different approaches have been proposed for designing an adaptive pair of sum and difference beams, such as the maximum-likelihood approach in [1], which yields a pair of beams that optimizes a selected angle estimator.

Rather than directly optimizing an angle estimator, however, it is possible to minimize the interference in the individual sum and difference output channels by employing linearly constrained optimization. Adaptive sum and difference beams are formed by applying sum and difference unity gain constraints at the look direction,

$$
\mathbf{w}_{\Sigma}^{H} \mathbf{v}_{\Sigma}=1, \quad \mathbf{w}_{\Delta}^{H} \mathbf{v}_{\Delta}=1,
$$

which, from (6), yields minimum variance (MV) sum and difference weights

$$
\mathbf{w}_{\Sigma}=\frac{\mathbf{R}_{\mathbf{x}}^{-1} \mathbf{v}_{\Sigma}}{\mathbf{v}_{\Sigma}^{H} \mathbf{R}_{\mathbf{x}}^{-1} \mathbf{v}_{\Sigma}}, \quad \mathbf{w}_{\Delta}=\frac{\mathbf{R}_{\mathbf{x}}^{-1} \mathbf{v}_{\Delta}}{\mathbf{v}_{\Delta}^{H} \mathbf{R}_{\mathbf{x}}^{-1} \mathbf{v}_{\Delta}} .
$$

Note that a difference processor can be obtained from the adaptive sum processor in (15) by differentiating the numerator and normalizing the resulting weight vector. If the sum 


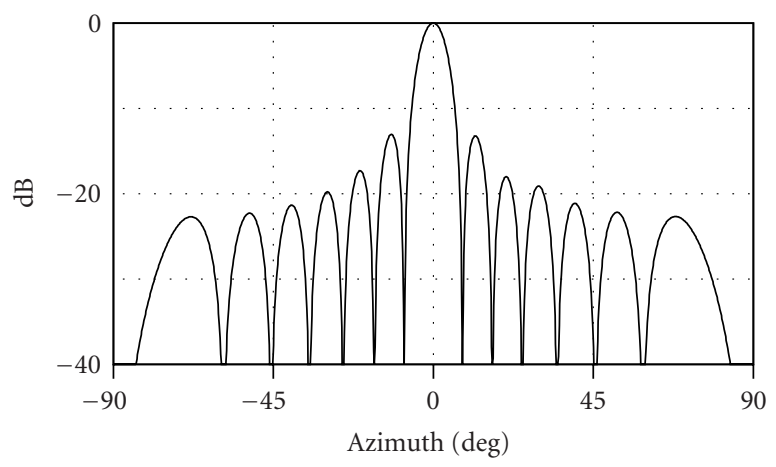

(a)

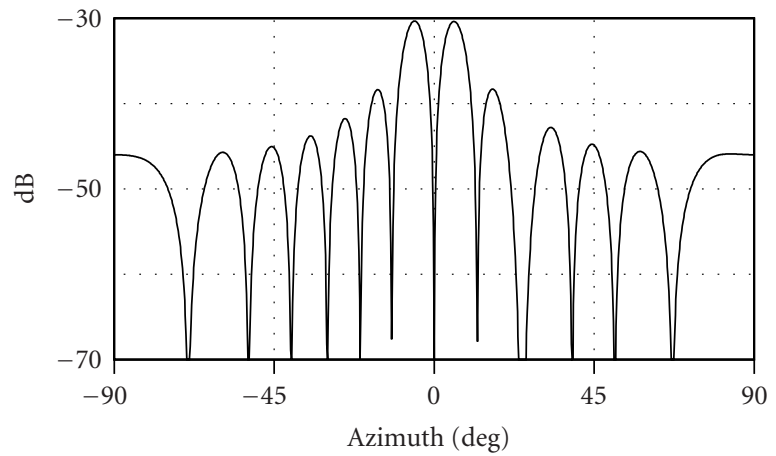

(b)

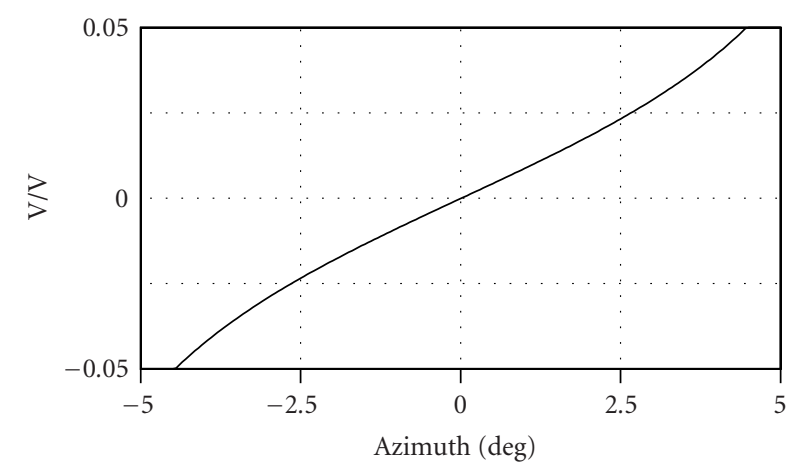

(c)

FIgUre 3: Monopulse (a) sum and (b) difference beams and (c) $\mathrm{MRC}$ for sidelobe jamming with a jammer at $25^{\circ}$.

and difference processors possess distorted mainbeams then a corresponding distorted MRC must be employed. A natural choice is to employ the definition for MRC provided earlier in (12) (i.e., the ratio of beam patterns). ${ }^{5}$ Thus, when no

\footnotetext{
${ }^{5}$ Although in the general case the ratio of beam patterns is a complex quantity, a complex MRC is avoided here. If a complex MRC is to be employed, the error voltage must be best-fitted to the MRC rather than inversemapped.
}

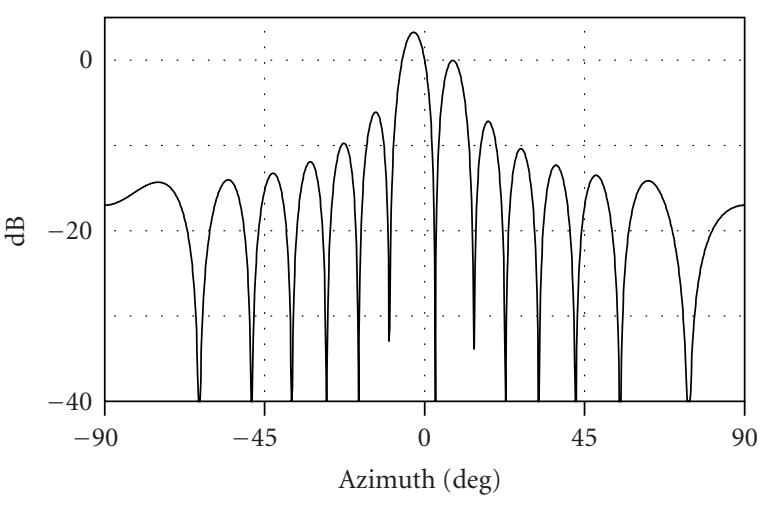

(a)

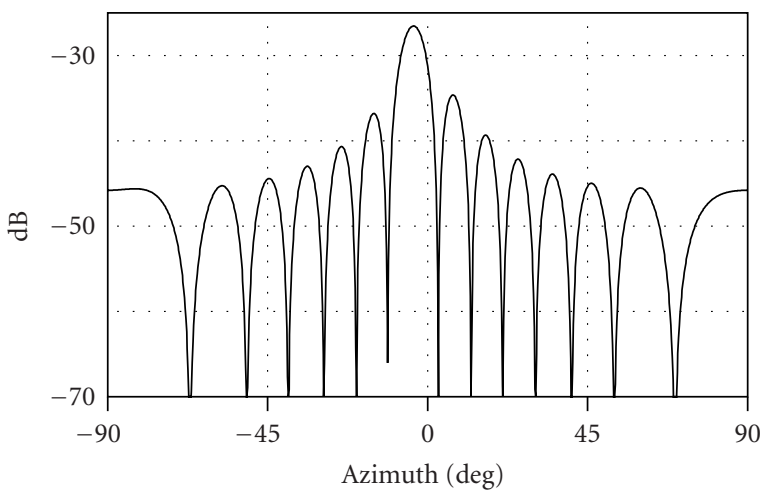

(b)

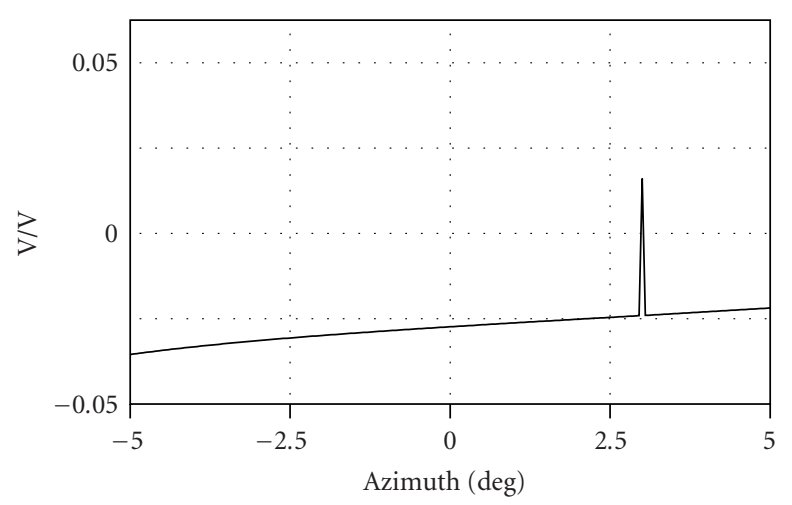

(c)

Figure 4: Monopulse (a) sum and (b) difference beams and (c) MRC for mainbeam jamming with a jammer at $3^{\circ}$.

interference is present then the angle estimates are exact, despite distortions that may be present in the beam patterns.

The technique works quite well for sidelobe jamming. Figure 3 illustrates the resulting sum and difference patterns as well as the MRC for a $60 \mathrm{~dB}$ sidelobe jammer at $25^{\circ}$. The resulting spatial null at $25^{\circ}$ is especially discernible in the difference beam. Otherwise the beams are well formed with no noticeable distortions.

The difficulty arises in the case of mainbeam jamming. Figure 4 shows spatially adaptive sum and difference patterns 
with a corresponding monopulse curve for a $60 \mathrm{~dB}$ mainbeam jammer at $3^{\circ}$. Distortions introduced by the jammer signal into the sum and difference beams are evident, in particular, the common null at $3^{\circ}$ that manifests itself as a singularity in the MRC. The distorted beams not only detract from the processor's angle estimation capability as evidenced by the flattened MRC, but also from the processor's robustness (i.e., susceptibility to false alarms).

\section{SPACE-TIME ADAPTIVE MONOPULSE}

As explained in the previous section, spatial adaptive monopulse processing does not always work well in a mainbeam jamming environment. However, we see from [2, 4 6] that space/fast-time (SFT) processing is able to provide substantial improvement in mainbeam jamming interference cancellation when TSI is present in the returns. The objective here is to develop a monopulse processor that employs SFT filtering to enhance angle estimation in much the same way that SFT filtering was employed in Section 2 to enhance interference mitigation. The approach taken entails

(i) generalizing the monopulse concept to an SFT processor;

(ii) exploring design considerations intended to overcome the limitations of spatial distortion discussed earlier with respect to spatial monopulse, and target spreading discussed in Section 2 with respect to SFT filters.

\subsection{Extending monopulse to SFT}

Recall that a monopulse system generates an error voltage signal, $\epsilon_{v}(l)$, and maps the error voltage to a corresponding angle measurement using a mapping function called a monopulse response curve (MRC) denoted by $\mathcal{M}$. For estimating angles in a single plane (i.e., azimuth or elevation), as will be done here, a single error voltage signal and MRC are required [10]. Care must be taken to ensure that $\mathcal{M}$ is invertible, otherwise the angle estimate for a given value of error voltage, $\epsilon_{v}$, may be ambiguous. Extension of spatial monopulse processing to SFT entails reinterpreting some of the spatial quantities defined in Section 3.

By definition, an SFT monopulse system has sum and difference filters that perform spatial as well as temporal filtering. The purpose of the temporal filtering is to rid the output signal of TSI and, possibly, mainbeam jamming interference. Sum and difference SFT processors are denoted by the $N T \times 1$ weight vectors $\mathbf{W}_{\Sigma}$ and $\mathbf{W}_{\Delta}$, respectively. Sum and difference outputs are given in terms of the respective processors,

$$
z_{\Sigma}(l)=\mathbf{W}_{\Sigma}^{H} \mathbf{X}(l), \quad z_{\Delta}(l)=\mathbf{W}_{\Delta}^{H} \mathbf{X}(l),
$$

where $\mathbf{X}(l)$ is the $N T \times 1$ SFT snapshot at time index $l$. The error voltage signal is, thus, a function of the ratio of SFT filtered outputs:

$$
\epsilon_{v}(l)=\mathfrak{R}\left\{\frac{z_{\Delta}(l)}{z_{\Sigma}(l)}\right\} .
$$

As before, the error voltage must convey purely directional information that is to be converted to angular form via a mapping function. Thus, the SFT sum and difference weights must be chosen so that the MRC is independent of the fast-time dimension. The MRC is independent of the fast-time dimension at the look direction when the sum and difference weights are formed using the same Frost constraint at the look direction. A particularly useful example is the range constraint which prevents target spreading. Target spreading is undesirable because it is detrimental to the sum beam detection.

The MRC, as defined earlier, is the ratio of spatial responses of the difference and sum processors within the mainbeam. Since the response of an SFT processor contains time dependencies a separate spatial response exists at each tap. However, given that range constraints are applied in such a manner that the target response is independent of fast-time, only the spatial response at tap, $T_{0}$, where a target look direction constraint has been applied (e.g., unity gain constraint), is of significance.

In general, the SFT response of a processor to a unit powered signal in a single range bin is defined as

$$
\boldsymbol{W}(\phi, \tau) \equiv \mathbf{W}^{H}\left[\delta_{T}(\tau) \otimes \mathbf{v}(\phi)\right]=\mathbf{W}(\tau)^{H} \mathbf{v}(\phi),
$$

where $\delta_{T}(\tau)=\left[\begin{array}{lll}\mathbf{0}_{1 \times \tau} & 1 & \mathbf{0}_{1 \times T-\tau-1}\end{array}\right]^{T}$ is a $T \times 1$ vector of zeros with a single 1 as its $\tau$ th component representing the target and $\mathbf{W}(\tau)$ denotes an $N \times 1$ vector comprised of the $N$ weights in $\mathbf{W}$ corresponding to tap $\tau$. The MRC is then the ratio of spatial responses at $T_{0}$ :

$$
\mathcal{M}(\phi) \equiv \mathfrak{R}\left\{\frac{\mathcal{W}_{\Delta}\left(\phi, T_{0}\right)}{\mathcal{W}_{\Sigma}\left(\phi, T_{0}\right)}\right\} .
$$

\subsection{Optimization criterion}

Adaptive monopulse algorithms have previously been developed using for example the maximum-likelihood (ML) technique [1]. ML, which minimizes the variance of the angle estimate error directly, may achieve true optimality given it is asymptotically unbiased. However, in this paper a constrained linear optimization technique is considered in the development of an SFT monopulse processor for the following reasons. In contrast to a maximum-likelihood (ML) approach, the use of linear constraints allows the designer to exercise a great deal of control over both the spatial and temporal behaviors of the SFT sum and difference processors, thus, assuring robustness by providing a means to avoid target spreading and other distorting effects. However, because angle estimation is not the criteria being optimized, a small but acceptable price may be paid in terms of angle estimation performance.

\subsection{Sum and difference filter design}

Having reinterpreted the MRC for SFT and selected an optimization criteria, the next step is to design sum and difference processors, $\mathbf{W}_{\Sigma}$ and $\mathbf{W}_{\Delta}$, that will not suffer from spatial distortions and yet provide adequate suppression of mainbeam jamming with minimal target spreading. The desired monopulse processor has the target response characteristics 


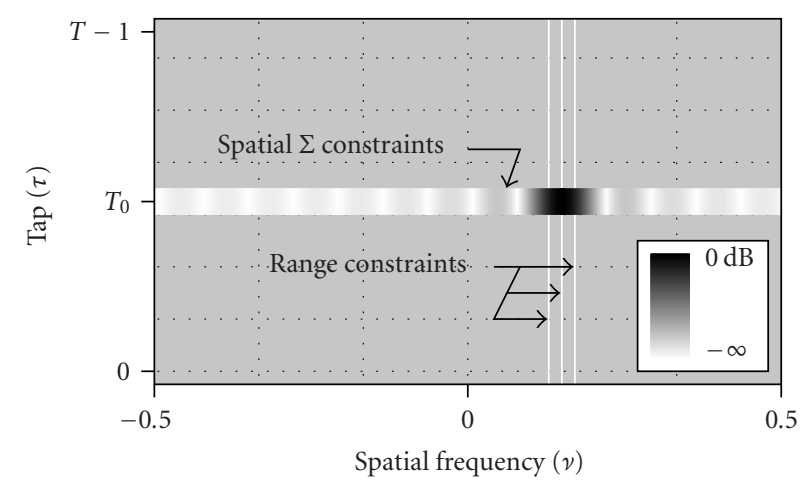

(a)

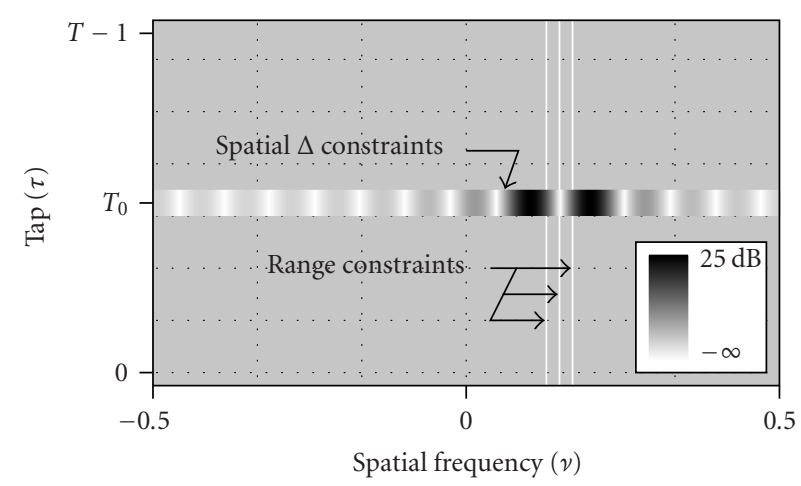

(b)

Figure 5: Constraint specifications for the space-time adaptive monopulse processor. (a) Sum processor. (b) Difference processor.

shown in Figure 5. The SFT processor considered is that of (6) with a set of constraints chosen to achieve these criteria. Of the $T$ taps in the processor, the $T_{0}$ th tap captures the target as shown in Figure 5.

To prevent target spreading in the look direction, range constraints are applied at the look direction $\left(\nu_{\ell}\right)$ for all taps except $T_{0}$ (shown as the center vertical white line in Figure 5). In general, however, it is not sufficient to apply one set of range constraints at the look direction. Since the detected target may have been detected anywhere within the mainbeam, it is advantageous to provide additional range constraints about the look direction at spatial frequencies, $v_{\ell} \pm 0.5 / \mathrm{N}$ (shown as additional vertical white lines in Figure 5). They do not ensure zero gain throughout the angular extent of the mainbeam but rather serve as "anchors" to keep the gain low in that region, and, as such, they serve to maintain independence of the MRC with respect to the fast-time dimension. The specified range constraints are implemented via a constraint matrix and vector:

$$
\mathbf{C}_{0}=\left[\begin{array}{cc}
\mathbf{I}_{T_{0}} & \mathbf{0}_{T_{0} \times T-T_{0}} \\
\mathbf{0}_{T-T_{0}-1 \times T_{0}+1} & \mathbf{I}_{T-T_{0}-1}
\end{array}\right] \otimes\left[\begin{array}{c}
\mathbf{v}\left(\nu_{\ell}-\frac{1}{2 N}\right)^{H} \\
\mathbf{v}\left(\nu_{\ell}\right)^{H} \\
\mathbf{v}\left(\nu_{\ell}+\frac{1}{2 N}\right)^{H}
\end{array}\right],
$$

where $\mathbf{0}_{n}$ is a vector of $n$ zeros.
Spatial response constraints (SRC) are defined for an expected target at tap $T_{0}$. Typically, a unity gain constraint is used, but, for the case of monopulse processing in mainbeam jamming, a more rigid set of constraints may be necessary to ensure a reliable and robust MRC. This requirement is met most stringently by forcing the processor to take the form of a conventional processor at tap $T_{0}$, in which case the spatial responses of the processors are identical to one of those shown in Figure $1 .^{6}$

This condition can be met by applying the constraint matrix and vector

$$
\begin{aligned}
& \mathbf{C}_{1}=\left[\begin{array}{lll}
\mathbf{0}_{1 \times T_{0}} & 1 & \mathbf{0}_{1 \times T-T_{0}-1}
\end{array}\right] \otimes \mathbf{I}_{\mathbf{N}}, \\
& \mathbf{c}_{1}= \begin{cases}\mathbf{v}_{\Sigma}, & \text { if sum processor, } \\
\mathbf{v}_{\Delta}, & \text { if difference processor. }\end{cases}
\end{aligned}
$$

The sum and difference steering weights at tap $T_{0}$ can be the steering vectors $\mathbf{v}_{\Sigma}$ and $\mathbf{v}_{\Delta}$ given in (8), or some other valid sum and difference pair. Although at first glance a conventional beam constraint would seem counterproductive, for it allows the mainbeam jammer to severely corrupt the output, recall, however, that the jammer cancellation is not to come from a spatial null, but rather from the TSI itself.

An alternative and simpler method for implementing the SRC is to apply a large degree of diagonal loading to the portion of the covariance matrix $\mathbf{R}_{\mathbf{X}}$ corresponding to $T_{0}$,

$$
\mathbf{R}_{\mathbf{X}} \longrightarrow \mathbf{R}_{\mathbf{X}}+\sigma_{d}^{2}\left[\delta_{T}\left(T_{0}\right) \cdot \delta_{T}\left(T_{0}\right)^{T}\right] \otimes \mathbf{I}_{N}
$$

along with a unity gain constraint at $T_{0}$,

$$
\mathbf{C}_{1}^{\text {alt }}=\left\{\begin{aligned}
& {\left[\delta_{T}\left(T_{0}\right) \otimes \mathbf{v}_{\Sigma}\right]^{H} } \text { if sum processor, } \\
& {\left[\delta_{T}\left(T_{0}\right) \otimes \mathbf{v}_{\Delta}\right]^{H} } \text { if difference processor, } \\
& \mathbf{c}_{1}^{\text {alt }}=1 .
\end{aligned}\right.
$$

Both approaches for implementing the SRC result in identical processors. In the first approach adaptation is suppressed at $T_{0}$ via constraints, whereas in the latter approach diagonal loading suppresses the adaptation at $T_{0}{ }^{7}$

Finally, the range constraints are grouped with the spatial response constraints giving the constraint matrix and vector

$$
\mathbf{C}=\left[\begin{array}{l}
\mathrm{C}_{0} \\
\mathrm{C}_{1}
\end{array}\right], \quad \mathbf{c}=\left[\begin{array}{l}
\mathrm{c}_{0} \\
\mathrm{c}_{1}
\end{array}\right] .
$$

The sum and difference processors are specified in terms of the design parameter $T_{0}$. The significance of $T_{0}$ is in the type of prediction that the resulting processor performs. In the derivations of $[2,3]$, a forward prediction filter is implemented by specifying that the target appear in the first tap

\footnotetext{
${ }^{6}$ Such a rigid set of constraints (i.e., which do not allow adaptivity to take place at $T_{0}$ ) exacts a heavy price in performance, as will be demonstrated. This point is discussed further in Section 6.

7 The latter approach has the advantage that through reduced diagonal loading the constraints may be "relaxed."
} 
(i.e., $T_{0}=0$ ). On the other hand, if the target is constrained to appear in the final tap (i.e., $T_{0}=T-1$ ), a backward prediction filter results. Results on experimental data verified that for monopulse processing a tap-centered configuration (i.e., $T_{0}=T / 2$ ) corresponding to a combination of forward and backward prediction is the best. The rationale is that components of the jamming multipath signals in the sidelobes arrive both before and after the corresponding mainbeam components. Although geometry dictates that the multipath should always arrive after the direct path jammer, the particular radar considered has $200 \mathrm{kHz}$ Gaussian front-end filters prior to a $1 \mathrm{MHz}$ sampler, which introduce artificial correlation between neighboring samples so that a back sample of TSI is correlated with a current jammer sample, and therefore useful in its prediction. In general, this reasoning applies if the degree of oversampling is moderate and only a small delay exists between the direct path jammer and TSI. In the opposite extreme (high oversampling and large delay between direct path and TSI) a forward prediction filter would be necessary.

In summary, extending monopulse to an SFT processor entails the following key steps:

(i) defining desired response characteristics for the sum and difference channels, so that the MRC remains independent of fast-time effects;

(ii) selecting an optimization criteria and solving for the sum and difference channel processors.

After achieving these goals, angle estimation proceeds in the same way as described in Section 3.

\section{SIMULATION RESULTS}

An evaluation of the new SFT monopulse concept is conducted on experimental dataset mmit004v1 containing a direct-path barrage noise jammer at $32^{\circ}$ and stationary TSI collected as part of the DARPA/Navy Mountaintop Program $[11,12]$. The radar employed is a 14-phase-center UHF phased array operating horizontally. These examples include a qualitative evaluation of the beam pattern response and $\mathrm{MRC}$, and a quantitative evaluation of angle estimation performance. Various design issues for SFT monopulse and their tradeoffs are considered as well.

\subsection{Beam pattern response}

Figure 6 illustrates sum and difference beam patterns, and MRC, for a spatially adaptive monopulse processor. The mainbeam is positioned about the look direction at $32^{\circ}$ as indicated by the dashed line. The jammer is colocated with the mainbeam. The high sidelobes seen in the $\Sigma$ beam pattern are typical of mainbeam jamming. Although such high sidelobes would be unacceptable in an actual radar processor implementation, when both the transmit and receive beam patterns are taken into consideration the sidelobes appear much lower. Therefore, the focus here is rather on the mainbeam which is positioned about the look direction. For the adaptive processor the mainbeam appears skewed, bulging to the

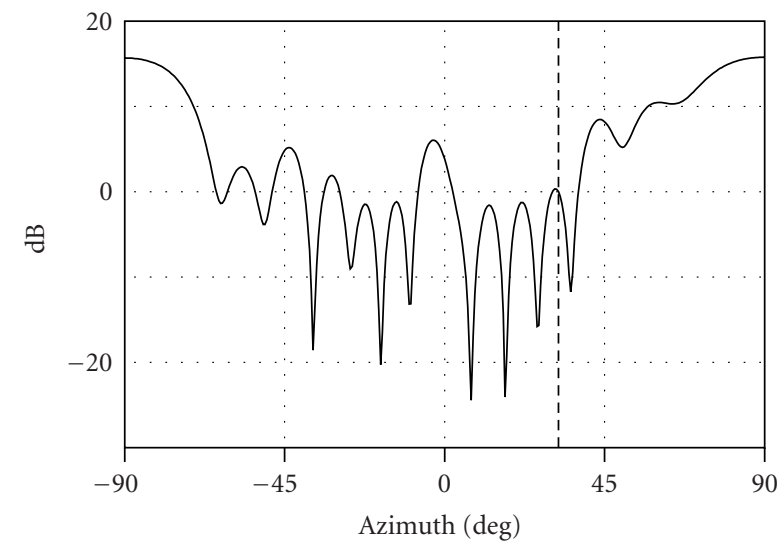

(a)

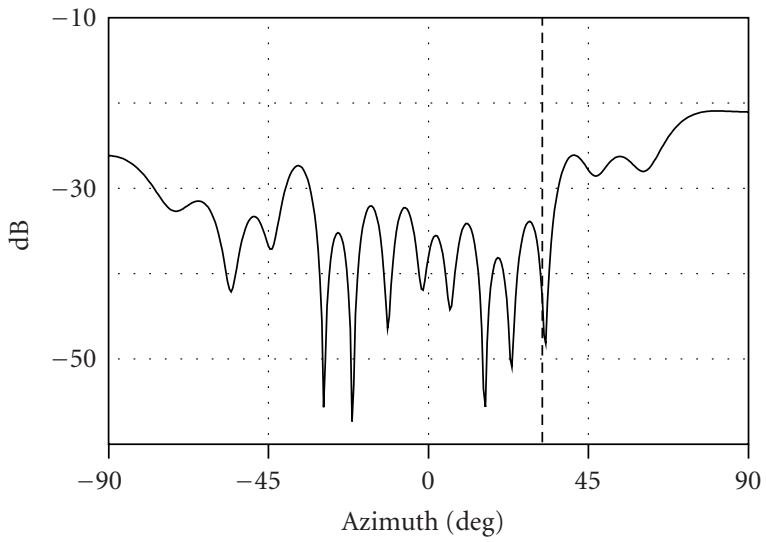

(b)

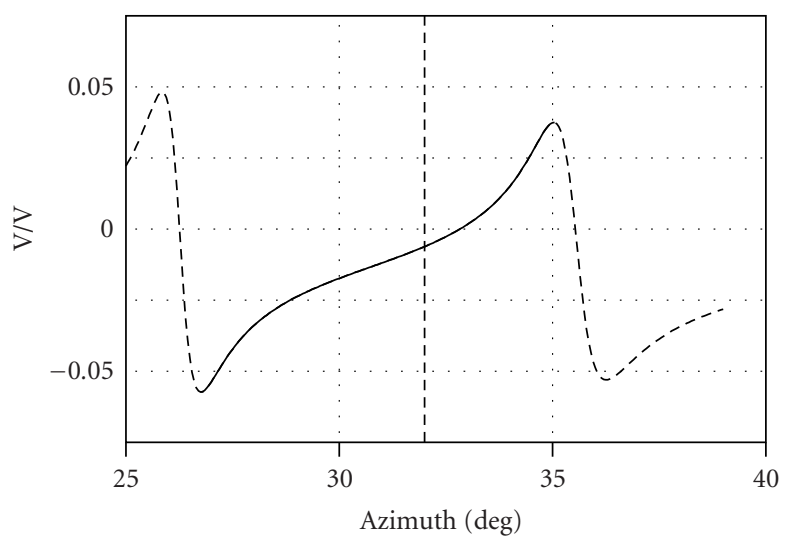

(c)

Figure 6: (a) Sum and (b) difference beam patterns, and (c) MRC, for a spatially adaptive monopulse processor.

left of the look direction. Likewise the resulting MRC is distorted and invertible only on a $8^{\circ}$ interval.

Illustrated in Figure 7 are both sum and difference beam patterns for a 20-tap, tap-centered monopulse processor. The resulting spatial beam pattern at $T_{0}=9$ is that of a conventional processor as dictated by the SRC of (20)-(23). 


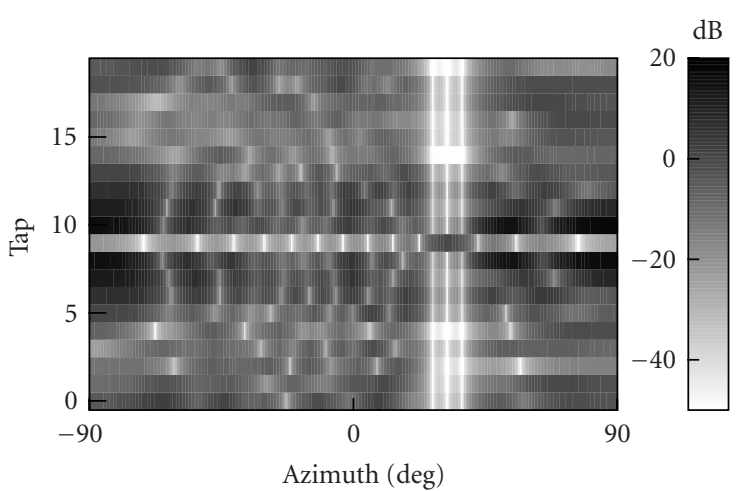

(a)

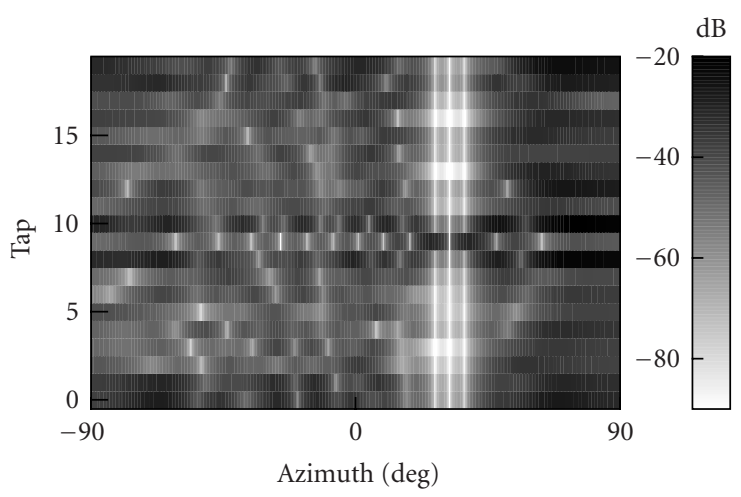

(b)

Figure 7: (a) Sum and (b) difference beam patterns for fully constrained SFT processor.

Additionally, target spreading is greatly reduced throughout the extent of the mainbeam as demonstrated by the deep nulls cutting across range. The worst target spreading artifacts appear near $T_{0}$ in-between range nulls at about $20 \mathrm{~dB}$ below peak target strength. However, most of the target spreading has been kept to $35 \mathrm{~dB}$ or more below peak target strength.

The undistorted response at $T_{0}$ comes at a cost. Sidelobe artifacts away from $T_{0}$ are significant, in particular those to the right of the mainbeam, one tap below and above $T_{0}$ (only the ones near the mainbeam concern us since those away from the mainbeam are significantly attenuated when considering the two-way pattern). The reason for the artifacts is that the processor must employ TSI and have it magnified significantly in order to predict and cancel the mainbeam jammer.

A second cost is paid in terms of performance. As will be demonstrated next, the 20-tap constrained processor yields similar angle estimation performance as the 1-tap spatially adaptive processor (discussed in Section 3.3). This is so since the constrained processor is unable to mitigate the mainbeam jammer directly (spatially), but rather through employing TSI to predict and cancel it. In short, what was lost in preserving the spatial response at the target cell of interest costs in taps.

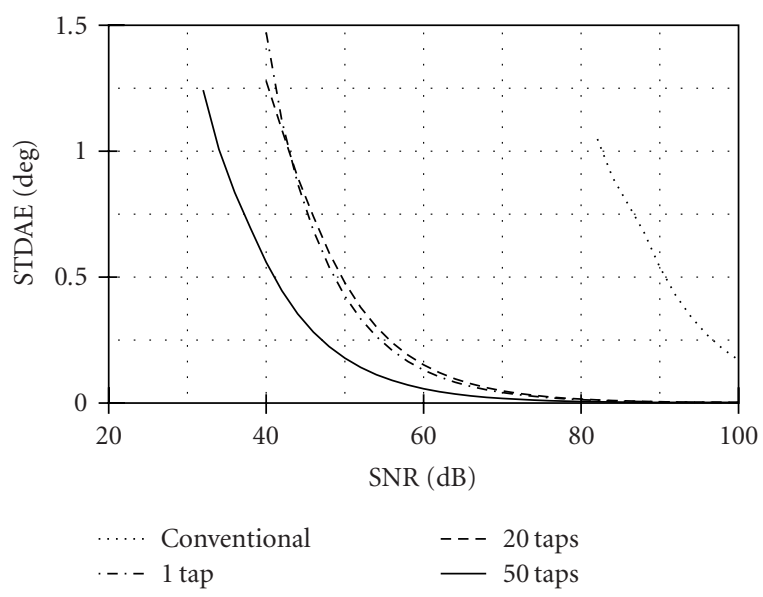

FIGURE 8: Angle estimation performance versus target SNR for four processors.

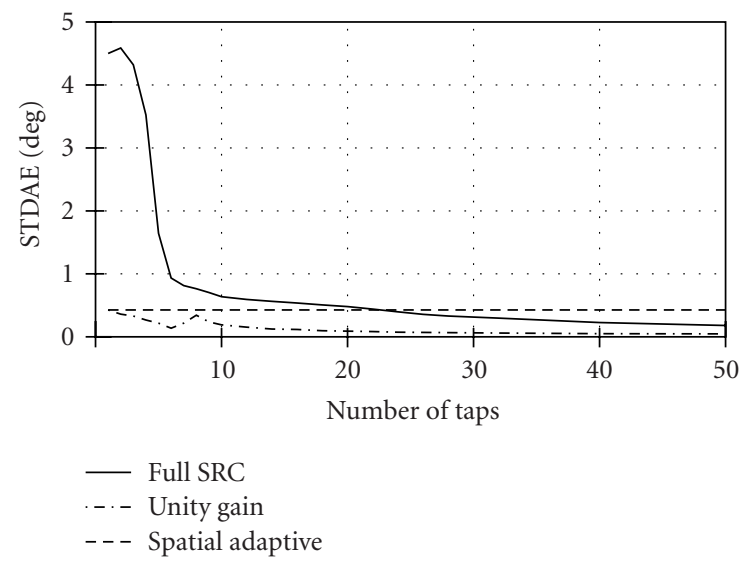

FIGURE 9: STDAE versus number of taps for fully constrained processor and unity gain SFT processor.

\subsection{Angle estimation performance}

The angle estimation performance of the 20- and 50-tap SFT processors is shown in Figure 8. Included for reference are the conventional processor and spatial adaptive processor. As mentioned earlier, the fully constrained SFT processor suffers from mitigation performance degradation in exchange for preservation of the spatial response at tap $T_{0}$. This is true for angle estimation performance as well, where it is noted that the 20-tap fully constrained processor performs similarly to the unity gain spatial processor.

\subsection{Tap analysis}

Figure 9 fixes the SNR at $50 \mathrm{~dB}$ and varies the number of taps from 1 to 50 for the fully constrained SFT processor and for the unity gain SFT processor. The fully constrained SFT processor utilizes a full set of SRCs whereas the unity 
gain SFT processor utilizes a single unity gain constraint. ${ }^{8}$ Both employ a tap-centered configuration with three sets of range constraints about the look direction. STDAE is plotted versus the number of taps in the processor. The solid and dash-dotted line curves indicate performance for the fully constrained and unity gain SFT processors, respectively. A horizontal dashed line indicates the performance of the spatially adaptive processor. The plot suggests that a 22-tap fully constrained SFT processor is necessary to achieve the same level of angle estimation performance as the spatially adaptive processor. As noted before, this is a necessary cost incurred for maintaining the spatial response at tap $T_{0}$ of the fully constrained processor. On the other hand, any number of taps in the unity gain constrained SFT processor improves on the spatial adaptive processor.

\subsection{Other results}

Similar results have been demonstrated for other MT datasets. The exceptions are MT datasets containing nonstationary TSI (i.e., resulting from a moving jammer and/or platform). The presence of nonstationary TSI makes it more difficult to predict the temporal structure of the jammer given that there are angle dependent Doppler shifts in the TSI returns. For reasonable performance in the presence of mainbeam jamming and nonstationary TSI, Doppler processing must be incorporated into the monopulse processor. One such algorithm is discussed in $[13,14]$.

\section{CONCLUSIONS}

The main innovation introduced in this paper is a method by which a monopulse processor is combined with an adaptive SFT processor to provide a precise angle tracking capability in the presence of TSI and mainbeam jamming. Key features of the new processor are a tap-centered configuration, extended range constraints, and spatial response constraints. The tap-centered configuration was best for the data investigated, but may not always be the best option (e.g., for large delay between the direct path and TSI signals). Range constraints in the look direction are intended to prevent spreading throughout the mainbeam, but the application of spatial constraints at $T_{0}$ imposes such a burden on the processor that target spreading into other taps becomes a problem. Therefore, additional range constraints about the look direction become necessary. An alternative approach to 3 sets of range constraints would be a single set of range constraints together with a set of slope constraints at taps others than $T_{0}$.

Spatial response constraints prevent distortions in the mainbeam. However, the spatial response constraints discussed here are too rigid than may be necessary. Complete relaxation of the constraints in the mainbeam resulted in the

\footnotetext{
8 The unity gain SFT processor has range constraints to avoid target spreading, but only a unity gain constraint at $T_{0}$. It is, thus, expected to perform better than both the fully constrained processor and a spatially adaptive processor. On the other hand, it suffers from spatial distortions of the beam patterns in the presence of mainbeam jamming.
}

unity gain SFT processor mentioned in Section 5.3, which significantly outperformed the fully constrained SFT processor as evidenced by Figure 9. The unity gain SFT processor, however, suffers from distorted and lopsided sum and difference beams as does the spatially adaptive processor. In practice, partial relaxation of the SRCs may be the best course. Relaxation of constraints maybe achieved through application of a unity gain constraint together with slope constraints in the mainbeam [8].

Previous work has shown no clear advantage in using different tap distributions. This is not the case for a monopulse SFT processor, where the necessity for a combination of forward and backward predictions was determined experimentally.

Although sufficient results have been presented to demonstrate the merit of SFT monopulse, the reader is referred to [13] for a more through investigation of this technique including treatment of the following issues:

(i) effects of constraint relaxation through reduced diagonal loading,

(ii) beamwidth reduction due to mainbeam distortions,

(iii) effect of including the transmit pattern on sidelobe artifacts.

\section{REFERENCES}

[1] R. C. Davis, L. E. Brennan, and L. S. Reed, "Angle estimation with adaptive arrays in external noise fields," IEEE Transactions on Aerospace and Electronic Systems, vol. 12, no. 2, pp. 179-186, 1976.

[2] S. M. Kogon, Adaptive array processing techniques for terrain scattered interference mitigation, Ph.D. thesis, Georgia Institute of Technology, Atlanta, Ga, USA, 1997.

[3] S. M. Kogon, D. B. Williams, and E. J. Holder, "Beamspace techniques for hot clutter cancellation," in Proceedings of IEEE International Conference on Acoustics, Speech, and Signal Processing (ICASSP '96), vol. 2, pp. 1177-1180, Atlanta, Ga, USA, May 1996.

[4] S. M. Kogon, E. J. Holder, and D. B. Williams, "Mainbeam jammer suppression using multipath returns," in Proceedings of 31st Asilomar Conference on Signals, Systems \& Computers, vol. 1, pp. 279-283, Pacific Grove, Calif, USA, November 1997.

[5] S. M. Kogon, E. J. Holder, and D. B. Williams, "On the use of terrain scattered interference for mainbeam jammer suppression," in Proceedings of 5th Annual Workshop on Adaptive Sensor Array Processing (ASAP '97), MIT Lincoln Laboratory, Lexington, Mass, USA, March 1997.

[6] S. M. Kogon, D. B. Williams, and E. J. Holder, "Exploiting coherent multipath for mainbeam jammer suppression," IEE Proceedings-Radar, Sonar and Navigation, vol. 145, no. 5, pp. 303-308, 1998.

[7] A. S. Paine, "Application of the minimum variance monopulse technique to space-time adaptive processing," in Proceedings of 2000 IEEE International Radar Conference, pp. 596-601, Alexandria, Va, USA, May 2000.

[8] U. Nickel, "Generalised monopulse estimation and its performance," in Proceedings of 3rd IEEE International Symposium on Signal Processing and Information Technology (ISSPIT '03), pp. 174-177, Darmstadt, Germany, December 2003. 
[9] O. L. Frost III, "An algorithm for linearly constrained adaptive array processing," Proceedings of the IEEE, vol. 60, no. 8, pp. 926-935, 1972.

[10] S. M. Sherman, Monopulse Principles and Techniques, Artech House, Norwood, Mass, USA, 1984.

[11] G. W. Titi and D. F. Marshall, "The ARPA/NAVY mountaintop program: adaptive signal processing for airborne early warning radar," in Proceedings of IEEE International Conference on Acoustics, Speech, and Signal Processing (ICASSP '96), vol. 2, pp. 1165-1168, Atlanta, Ga, USA, May 1996.

[12] USAF Rome Laboratory, Mountaintop Program Summit Data: ASAP 1995 Data Release, March 1995.

[13] Y. Seliktar, Space-time adaptive monopulse processing, Ph.D. thesis, Georgia Institute of Technology, Atlanta, Ga, USA, 1998.

[14] Y. Seliktar, D. B. Williams, and E. J. Holder, "Adaptive monopulse processing of monostatic clutter and coherent interference in the presence of mainbeam jamming," in Proceedings of 32nd Asilomar Conference on Signals, Systems \& Computers, vol. 2, pp. 1517-1521, Pacific Grove, Calif, USA, November 1998.

Yaron Seliktar was born in Glasgow, Scotland, on September 22, 1969. He received his B.S. degree in electrical engineering from Drexel University in 1992, an M.S. degree in electrical engineering from the Georgia Institute of Technology in September 1993, and a Ph.D. degree in electrical engineering from the Georgia Institute of Technology in March 1999. Concurrently, Dr. Seliktar held an appointment of Re-

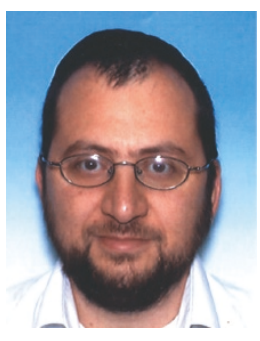
search Assistant from September 1994 to September 1996 in the Digital Signal Processing Laboratory at the Georgia Institute of Technology, and in September 1996 he was appointed Research Assistant at the Georgia Tech Research Institute (GTRI), in the Sensors and Electromagnetic Applications Laboratory. In February 1999 he joined the Electronic Sensors and Systems Division of Norden Systems Northrop Grumman at Norwalk Connecticut, where he held an appointment as a Ph.D. Research Engineer. In October 2003 he joined the Academic Faculty of the Jerusalem College of Engineering, and has held this position to date. Seliktar's research interests include statistical signal processing and adaptive spacetime array processing, and their application to radar signal processing.

Douglas B. Williams received the B.S.E.E., M.S., and Ph.D. degrees in electrical and computer engineering from Rice University, Houston, Texas, in 1984, 1987, and 1989, respectively. In 1989, he joined the faculty of the School of Electrical and Computer Engineering at the Georgia Institute of Technology, Atlanta, Georgia, where he is currently an Associate Professor and Associate Chair for Undergraduate Affairs. There he

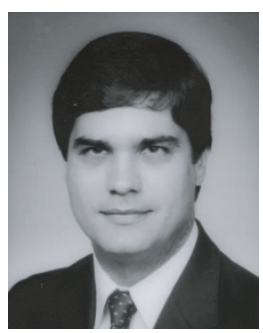
is also affiliated to the Center for Signal and Image Processing and the Arbutus Center for Distributed Engineering Education. Dr. Williams has served as an Associate Editor of the IEEE Transactions on Signal Processing and the EURASIP Journal on Applied Signal Processing. He is a Member of the IEEE Signal Processing Society's Board of Governors, SPTM Technical Committee, and Education Technical Committee. He was on the Conference Committee for the 1996 International Conference on Acoustics, Speech, and Signal Processing and was Cochair of the 2002 IEEE DSP and Signal Processing Education Workshops. Dr. Williams was a coeditor of the Digital Signal Processing Handbook published in 1998 by CRC Press and IEEE Press. He is a Member of the Tau Beta Pi, Eta Kappa $\mathrm{Nu}$, and Phi Beta Kappa honor societies.

E. Jeff Holder is currently Chief Scientist and Principal Research Scientist in the Sensors and Electromagnetic Applications Laboratory of the Georgia Tech Research Institute, Georgia Institute of Technology, where he is also an Adjunct Professor in the School of Electrical and Computer Engineering. He received the B.A. degree in mathematics from Florida State University in 1969, the J.D. degree in law from the University of Mississippi in 1973, and the M.A. and Ph.D. degrees in mathematical physics from Duke University in 1978 and 1980. In 1990, Dr. Holder received the GTRI Laboratory Outstanding Research Award, and he is a member of IEEE and Sigma Xi. He is currently directing an effort to design a weapon/sensor architecture to engage rockets, artillery, and mortars for the US Army. He has directed numerous radar programs at GTRI including the SWORD/E-STRIKE Interferometric Radar Program and the Multi-Mission Radar Program where he was responsible for evaluating the hardware design and system performance. His research interests include multiresolution algorithm, optimal estimation and guidance, spectral estimation, and adaptive array processing. Dr. Holder has directed work developing tracking filters for radar and sonar, directed a project to design and fabricate a bistatic/digital beamforming array, and directed projects to develop optimal STAP algorithms for angle estimation and jammer cancellation. Dr. Holder has published over 60 technical papers, including topics in array calibration and on the effects of correlated noise on radar tracking performance. He also developed optimal polarization technologies for target detection in clutter and troposheric effect mitigation for low elevation target detection. He currently teaches courses on optimal target tracking and adaptive digital beamforming at Georgia Tech and has published numerous papers in the area of radar design and processing as well as guidance and control. 